SELECTED PAPER AT NCSP'21

\title{
Tomato Recognition Algorithm and Grasping Mechanism for Automation of Tomato Harvesting in Facility Cultivation
}

\author{
Suguru Uramoto, Hiroshi Suzuki, Akinobu Kuwahara, \\ Takahiro Kitajima and Takashi Yasuno \\ Graduate Schools of Sciences and Technology for Innovation, Tokushima University \\ 2-1 Minami-Josanjima, Tokushima 770-8506, Japan \\ E-mail: $\{$ uramoto-s, kitajima-t $\} @$ ee.tokushima-u.ac.jp \\ $\{$ suzuki.hiroshi, yasuno.takashi\}@ tokushima-u.ac.jp
}

\begin{abstract}
In this paper, we describe a tomato recognition algorithm and a fruit grasping mechanism for a tomato-harvesting robot that is designed to operate in a horticultural facility. In the tomato recognition algorithm, image processing is performed on the color images captured by a depth camera to detect red ripe large tomato and oval mini-tomato fruits. Then, the spatial coordinates of the fruit center and the diameter of the fruit are calculated using the depth information acquired by the depth camera. For the grasping mechanism, we designed and fabricated a mechanism for grasping an oval mini-tomato, which is necessary in the harvesting process. Experiments using these systems were conducted in a horticultural facility where tomatoes are grown, and the performances of both the tomato recognition and grasping motions were verified.
\end{abstract}

\section{Introduction}

In recent years, consumers in Japan have tended to choose higher quality produce, and agricultural businesses are reguared to maintain high quality and yield in their production. Large-scale facility cultivation is attracting attention and being introduced to enable higher efficiency and productivity in agriculture. On the other hand, Japanese agriculture faces serious problems related to labor shortages, such as a decline in the number of agricultural workers, an increase in the number of elderly owners, and a shortage of successors. As the population of Japan has been declining in recent years, these labor shortage problems are expected to become more serious in the future. In order to improve productivity and reduce the workload, many companies and research institutes are researching and developing agricultural robots.

In this study, we focused on the harvesting process of tomatoes from among agricultural crops. In Japan, about $80 \%$ of the distributed tomatoes are grown by facility cultivation. In the case of horticulture, where cultivation is conducted in a similar environment with minimal influence of weather, it is considered easier to introduce automatic machines such as agricultural robots. In addition, harvesting work in tomato cultivation accounts for about $35 \%$ of labor time, which is the second longest work time after management work [1]. On the basis of the above, we thought that automating the tomato harvesting process would help solve the labor shortage. To automate the harvesting process, a mobile robot that can move autonomously in the horticultural facility, a tomato recognition algorithm that can search the location and position of red ripe tomatoes on robot coordination, and a robot arm able for harvesting are needed to approach and grasp the detected tomatoes.

In this paper, we describe the tomato recognition algorithm and the grasping mechanism to grasp tomatoes during harvesting, which are necessary for the tomato-harvesting robot to operate in a horticultural facility. We also present several experimental results of using the developed SCARA-type robot arm.

\section{Tomato Recognition Algorithm}

To enable tomato harvesting by the robot, it is necessary to recognize the tomatoes within the camera viewing angle and to measure the distance and direction to the recognized tomatoes as accurately as possible. Tomatoes ripen to red at the same time as other fruits in the bunch, so tomato recognition must recognize multiple fruits individually. Therefore, we use image processing of the color image and depth information captured by the depth camera to recognize tomatoes and detect their spatial coordinates. Table 1 shows the specifications of the tested depth camera used in tomato recognition.

Table 1: Realsense L515

\begin{tabular}{c|c}
\hline RGB frame resolution & $1920 \times 1080$ \\
\hline Depth output resolution & $1024 \times 768$ \\
\hline Depth ideal range & $0.25 \mathrm{~m}$ to $9 \mathrm{~m}$ \\
\hline Depth technology & LiDAR \\
\hline Use environment & Indoor \\
\hline
\end{tabular}


In this study, in order to not interfere with the work of farmers, the tomato harvesting robot works at night. Therefore, tomato recognition is required to be performed under LED illumination. In addition, there is the advantage of reducing the distance noise of a depth camera compared with under daylight illumination, since the depth camera also detects infrared light. However, since the light intensity of LED illumination is weaker than that of daylight, it is necessary to verify whether tomatoes can actually be detected under LED illumination.

\subsection{Tomato recognition}

In tomato recognition by image processing, color images captured by a depth camera are used and images are processed by OpenCV. [2] The flow of tomato recognition by image processing is shown in Fig. 1, and each process is detailed below.

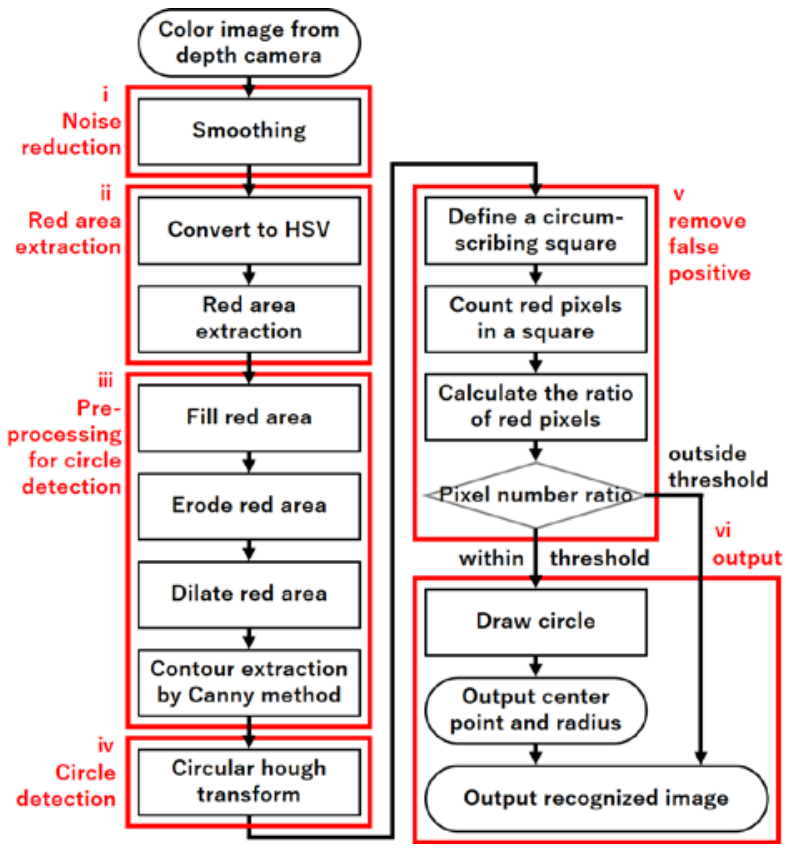

Figure 1: Flow chart of image prosessing for tomato detection

i. To reduce noise in order to extract the red area, the captured color image (Fig. 2(a)) is smoothed using a Gaussian filter.

ii. Convert RGB color space to HSV color space and extract the red area.

iii. To eliminate false positives in circle detection, fill, shrink, and expand the red area. Figure 2(b) shows the red region image after processing.

iv. To detect circles in the red region image, contour detection by the Canny method is performed. Detect a circle by circular Hough transform on a contour image. Figure 2(c) shows a color image with the detected circles superposed.

v. To remove false positives, the detection regions are judged using the proportion of red pixels in the circumscribed square of the detection circle. In this case, the regions where the red pixel proportion is $50 \%$ or more are judged to be those of tomatoes.

vi. Finally, an image of the recognition circle drawn on a color image, and the center coordinates and radius information of the recognized circle are output. Figure 2(d) shows the recognized image.

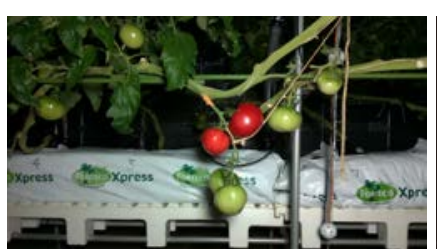

(a) Original color image

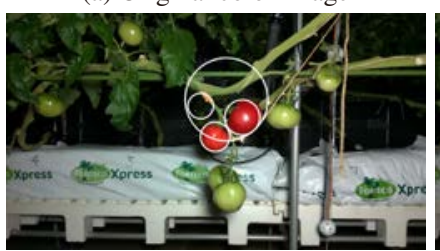

(c) Circular Hough transform

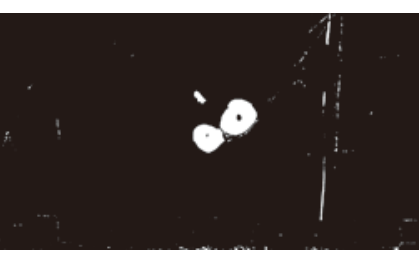

(b) Red area extraction

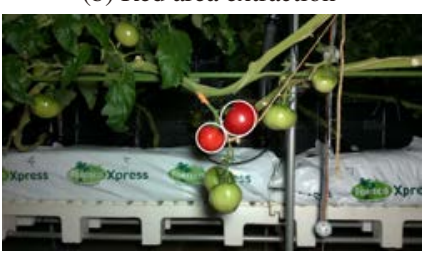

(d) Recognition image
Figure 2: Example of image processing results

\subsection{Detection of tomato}

In order to obtain the tomato position on the robot coordinate, the spatial coordinates of the center of the tomato are caluculated from the recognized circle and the depth information obtained by the depth camera. Figure 3 shows an example of a depth image acquired by the depth camera. The flow chart of detecting tomato coordinates from depth information is shown in Fig. 4.

i. Obtain the depth at the center coordinate of the recognized circle from the depth information. Partial coordinates are calculated from the depth information by mapping the angle of view of the RGB sensor to that of the depth sensor.

ii. In the same way, the depth and spatial coordinates are calculated at the coordinate that is shifted by an amount equal to the radius from the center of the recognized circle. The actual diameter is calculated based on the spatial coordinates of the tomato center and the spatial coordinates shifted by on amount equal to the radius. 
iii. To remove detected false positive tomatoes, determine whether the diameter of tomatoes is within the range of 30 to $100 \mathrm{~mm}$.

iv. If the detected circle is determined to be a tomato, draw the circle in the color image and output the spatial coordinates of the tomato center.

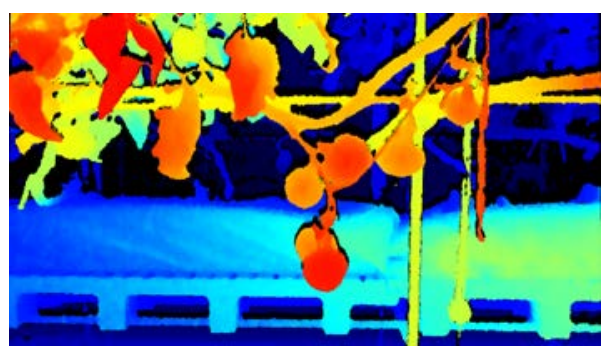

Figure 3: Example of depth image

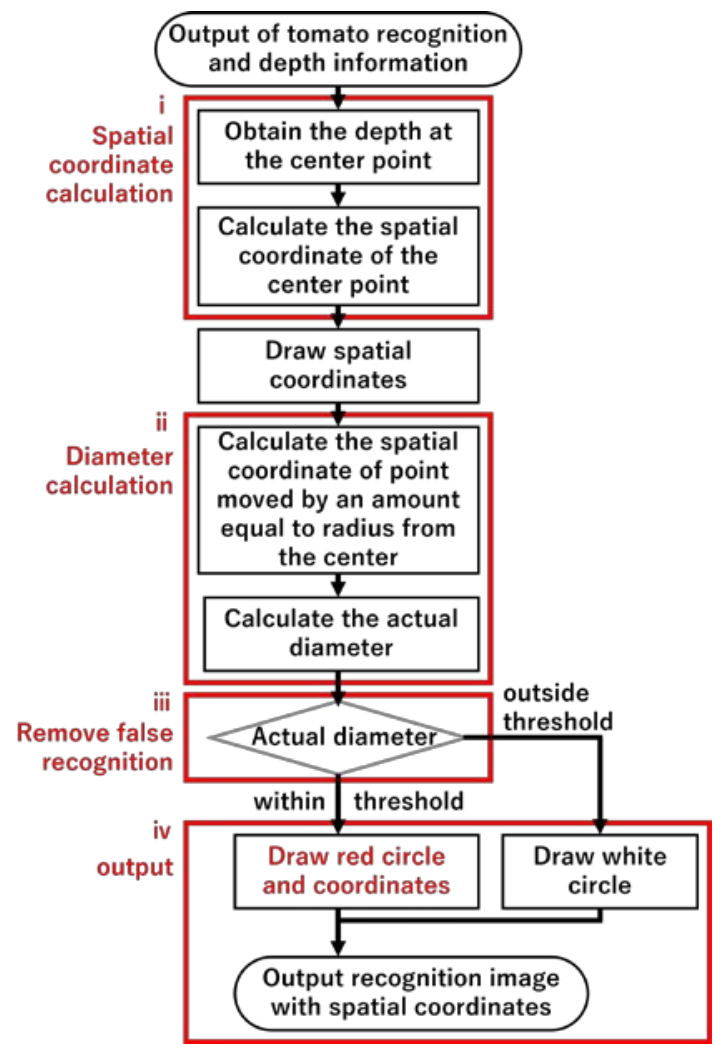

Figure 4: Flow chart of detecting tomato coordinates

\subsection{Tomato recognition results}

Figures 5(a) and 5(b) show recognition results of the large tomatoes and oval mini-tomatoes using the proposed tomato recognition algorithm.

In Fig. 5(a), four tomatoes (two on the front growing bed and two on the next lane) are recognized correctly. The tomatoes on the next seem to be illuminated with insufficient, but

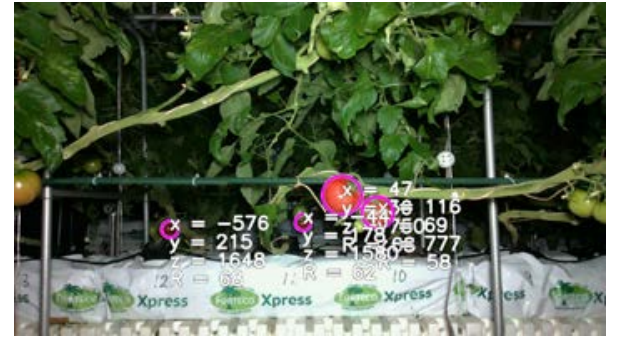

(a) Recognition result1

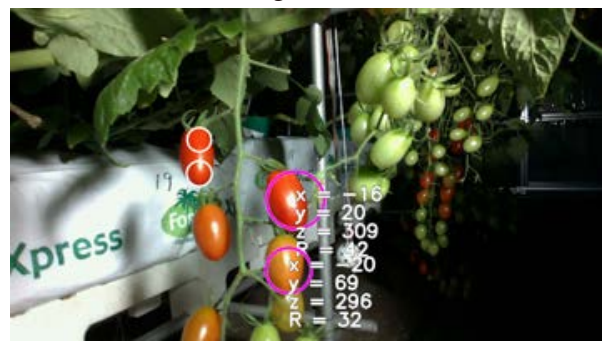

(b) Recognition result2

Figure 5: Recognition results

the spatial coordinates can be correctly obtained. In Fig. 5(b), two oval mini-tomatoes in the center of the image are recognized, but the center of the recognition circle does not match to the center of the fruit. In the case of the tomato on the left side of the image, two circles were detected in the same fruit and this fruit was not recognized successfully as one tomato. This is because the oval mini-tomato was detected as two circles with diameters of $30 \mathrm{~mm}$ or less.

These results indicate that tomato recognition is possible even at night, when there is insufficient ambient light. On the other hand, it was found that tomato recognition by circle detection could be applied to large tomatoes that were close to a circle in shape, but it was difficult to apply to oval minitomatoes.

\section{Grasping Mechanism Using Robot Arm}

Tomato harvesting requires a mechanism to separate the fruit from the bunch and to grasp and transport the fruit. In Japan, the commercial value of a tomato fruit is significantly reduced if it does not have a stalk. Therefore, it is necessary to avoid placing a heavy load on the fruit and the stems during harvesting. Farmers separate the fruit from the bunches by twisting the fruit while holding the stems with their fingertips, leaving the stems intact. In order for the machine to perform such an operation, it is required to detect the stem leading from the bunch to the fruit accurately. As a preliminary step to this stem detection, we thought it necessary to stabilize the fruits and stems without placing a large load on them. Therefore we designed and fabricated a grasping mechanism capable of this task. 


\subsection{Structure of the gripping mechanism}

The 3D model of our designed grasping mechanism using a SCARA-type robot arm is shown in Figs. 6(a) and 6(b). At the tip of the grasping part, an end-effector is capable of the basic function of opening and closing two fingers at the same time by rotating a servomotor to grasp the tomato. Since the grasping manipulator must support the weight of the harvested tomato, a horizontal articulated arm (SCARA-type) mechanism is used to reduce the overall weight and power consumption compared with a vertical arm. The lengths between the axes $\left(L_{1}, L_{2}\right.$, and $\left.L_{3}\right)$ are $250 \mathrm{~mm}$, and $L_{4}$ is 95 $\mathrm{mm}$. When the target position of the grasping end-effector $(\mathrm{x}, \mathrm{y})[\mathrm{mm}]$ is calculated, the target angle of each joint servo motor can be expressed by the following equation based on inverse kinematics.

$$
\begin{aligned}
\theta_{1}= & \cos ^{-1} \frac{\left(x+L_{4}\right)^{2}+\left(y-L_{3}\right)^{2}+L_{1}^{2}-L_{2}^{2}}{2 L_{1} \sqrt{\left(x+L_{4}\right)^{2}+\left(y-L_{3}\right)^{2}}} \\
& +\tan ^{-1} \frac{y-L_{3}}{x+L_{4}} \\
\theta_{2}= & \cos ^{-1} \frac{-\left(x+L_{4}\right)^{2}-\left(y-L_{3}\right)^{2}+L_{1}^{2}-L_{2}^{2}}{2 L_{1} L_{2}} \\
\theta_{3}= & \pi-\theta_{1}+\theta_{2} \\
\theta_{4}= & \frac{\pi}{2}
\end{aligned}
$$

Each joint is controlled by point-to-point control.

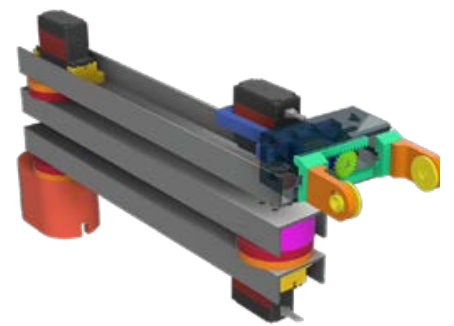

(a) $3 \mathrm{D}$ view

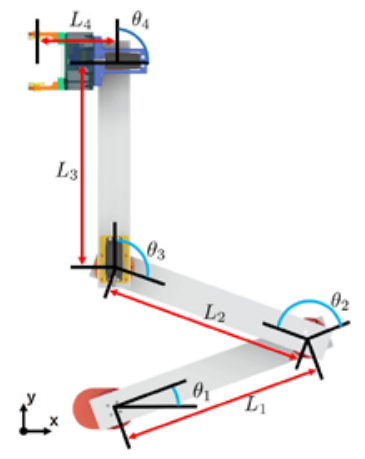

(b) Top view
Figure 6: Model of the grasping mechanism

\subsection{Experimented results}

Using the results of tomato recognition, we verified the operation of the grasping mechanism for oval mini-tomatoes. For the verification of the operation, the height of the grasping mechanism and the tomato were matched.

Figures 7 (a) to $7(d)$ show the approaching and grasping motions from the initial position to grasping. Position control on the X-Y coordinate system was successful with sufficient accuracy to grasp the tomato. However, owing to insufficient

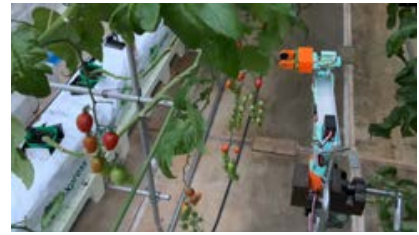

(a) Initial state

(c) Standby for grasping

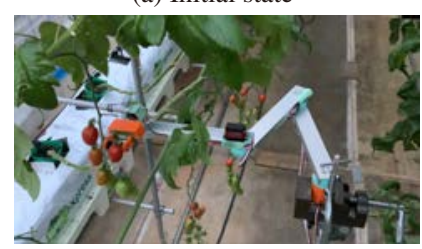

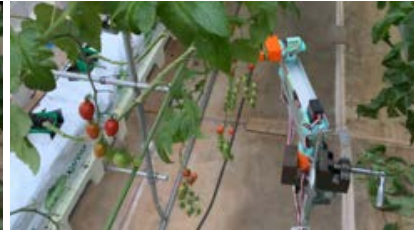

(b) Approaching

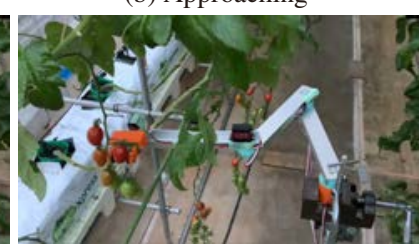

(d) Grasping
Figure 7: Results of approaching and grasping motions

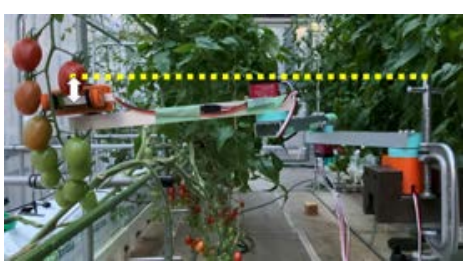

Figure 8: Deflection of arm

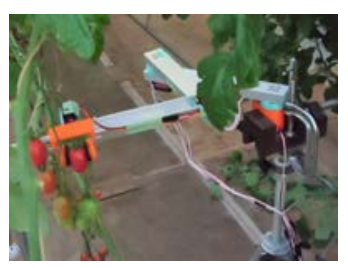

Figure 9: Approaching rigidity of the SCARA robot arm, a vertical axis position error of about $20 \mathrm{~mm}$ occurred as shown in Fig. 8. After adjusting the height of the tomato and the arm to account for the deflection of the arm, the tomato could be grasped, as shown in Fig. 9.

\section{Conclusions}

We described the tomato recognition algorithm and grasping mechanism, and verified their performances in a horticultural facility where tomatoes are grown. As a result, by the tomato recognition algorithm, both large tomatoes and some oval mini-tomatoes were successfully recognized and the spatial coordinates of the tomato center were obtained. In the grasping mechanism, the grasping motion was performed by controlling the position of the arm. Future tasks are to develop on ellipse detection algorithm for tomato recognition, and to redesign and fabricate a more rigid robot arm to improve the motion control accuracy.

\section{References}

[1] Ministry of Agriculture, Forestry and Fisheries: Large tomatoes management of institutional vegetable crops, Management Statistics by Commodity, Government Statistical Office (e-Stat), 2007.

[2] S. Uramoto, H. Suzuki, T. Kitajima, A. Kuwahara and T. Yasuno: Fruit recognition algorithm using depth camera for tomato harvesting robot, SJCIEE pp.13-14, 2020. 